\title{
The power supplies for the glow discharge electrodes in
}

\author{
Wendelstein 7-X
}

\author{
Thomas Rummel $^{\mathrm{a}}$, Frank Füllenbach ${ }^{\mathrm{a}}$, Günther Böhm ${ }^{\mathrm{b}}$, Wolfgang Kaesler ${ }^{\mathrm{b}}$, Rainer Burek ${ }^{\mathrm{b}}$, Steffen \\ Pingel $^{\mathrm{a}}$, Anett Spring ${ }^{\mathrm{a}}$, Jörg Schacht ${ }^{\mathrm{a}}$, Andreas Wölk ${ }^{\mathrm{a}}$ \\ ${ }^{a}$ Max-Planck-Institut für Plasmaphysik, Euratom Association, Wendelsteinstr. 1, D-17491 Greifswald, Germany \\ ${ }^{b}$ PPT Puls-Plasmatechnik GmbH, Feldstr. 56, D-44141 Dortmund, Germany
}

The conditioning of the Wendelstein 7-X (W7-X) plasma vessel will be done by glow discharges. Ten electrodes are placed inside of the vessel. Due to the three dimensional geometry the conditions for the ignition and the stabilization of a glow discharge can vary from one electrode to the other electrode. Therefore, ten individual power supplies with maximum ratings of $3 \mathrm{kV}$ and $3 \mathrm{~A}$ will be used. The ten power supplies are individually controlled by a PLC based control system. This control system is a major part of the overall glow discharge system, because it has to allow the combination of two or more power supplies to groups, which can be controlled in a similar way. The glow discharge is planned to run up to a week in steady state mode. This requires a sophisticated monitoring system of the parameters of the power supplies and the implementation of a proper matrix of reaction after failures. One aim of the W7-X control system is the remote control of all activities from the W7-X main control room. The glow discharge power supply control has to allow such operation and special procedures for exchange of control rights were implemented. The power supply system was developed, manufactured installed and tested in collaboration between IPP and PPT.

Keywords: Wendelstein 7-X, power supply, control system, glow discharge cleaning

\section{Introduction}

The stellarator fusion experiment Wendelstein 7-X (W7-X) is presently constructed at the Max-Planck-Institut für Plasmaphysik (IPP) in Greifswald, Germany. The basic machine consists mainly of the outer vessel, the superconducting coil system with its supports and the plasma vessel with its inventory. The plasma vessel is made of stainless steel with a weight of 30 tons, an outer diameter of approximately 12 meters and a surface of about 200 square meters, covering a volume of 110 cubic meters. The shape of the vessel follows the three dimensional shape of the plasma. The cross section varies ten times between a triangular cross section and a bean like cross section.

The conditioning of the plasma vessel during the start-up and after breaks will be done by glow discharges. Ten electrodes are placed inside of the vessel. One polarity is the glow discharge electrode and the vessel acts as the cathode. The conditions for the ignition and the stabilization of a glow discharge can vary from one electrode to the other electrode. It was therefore decided to supply each electrode by one independent power supply. The ten power supplies are individually controlled by a PLC based control system. This control system is a major part of the overall glow discharge system, because it has to combine all of the power supplies in order to control them in a similar way by the W7-X main control. The glow discharge is planned to run in steady state mode. This requires a sophisticated monitoring system of the parameters of the power supplies and the implementation of a proper matrix of reaction after failures. This feature is a second major part of the control system. One aim of the Wendelstein 7-X control system is the remote control of all activities from the W7-X main control room. The glow discharge power supply control has to allow such operation and special procedures for exchange of control rights were implemented. Another main part of the system is a test load which allows a test of the output parameters voltage, current and power of a converter independent from the W7-X.

In the following sections the main parts of the power supply system will be described.

\section{Main components}

\subsection{AC/DC converters}

The requirements of the converters were derived from a series of glow discharge experiments at a vacuum vessel with a W7-X like geometry [1]. For the ignition of the glow discharge a voltage up to $2.5 \mathrm{kV}$ is necessary and during the discharge currents of up to $3 \mathrm{~A}$ have to be delivered. The control principle of the output parameter control has to be changeable from a voltage regulation during the ignition phase to a current regulation during the discharge phase. More general requirements are low power losses, short-circuit-proofs, reactions times of less than 10 milli seconds. The usage of industrial products saves development costs and time. Therefore the type MCA3000-3000 from FuG Elektronik 
$\mathrm{GmbH}$, Rosenheim, Germany was selected. These power supplies have the capability of auto ranging which makes them rather small compared to a power supply that delivers to $3 \mathrm{kV}$ at $3 \mathrm{~A}$ which would result in a $9 \mathrm{~kW}$ unit consuming much more space. The given converter by FUG is limited to $3 \mathrm{~kW}$ but allows the mentioned above voltage and current control scenarios for the ignition and discharge, because the voltage during the discharge is about 300 to $500 \mathrm{~V}$. Only a small modification has to be made by introducing a series inductance of $26 \mathrm{mH}$ in the output line to stabilize the current regulation during the discharge. Main parameters of a converter are listed in table 1:

Table 1: main parameters of a converter

\begin{tabular}{cc}
\hline Designation & Value \\
\hline Input voltage & $400 \mathrm{~V}+/-10 \%$ \\
Max. Output voltage & $3 \mathrm{kV}$ \\
Max. d.c. Output current & $3 \mathrm{~A}$ \\
Max. Output power & $3 \mathrm{~kW}$ \\
Deviation from reference value & $<2 \times 10^{-4}$ \\
efficiency & $85 \%$ \\
interfaces & Profibus DP \\
System & 19 inch \\
\hline
\end{tabular}

The ten converters are located in three 19 inch racks, one rack contains four converters and the other two racks are equipped with three converters each. All racks are air cooled by fans, situated into the roof of the racks. Fig. 1 gives an impression of a rack.

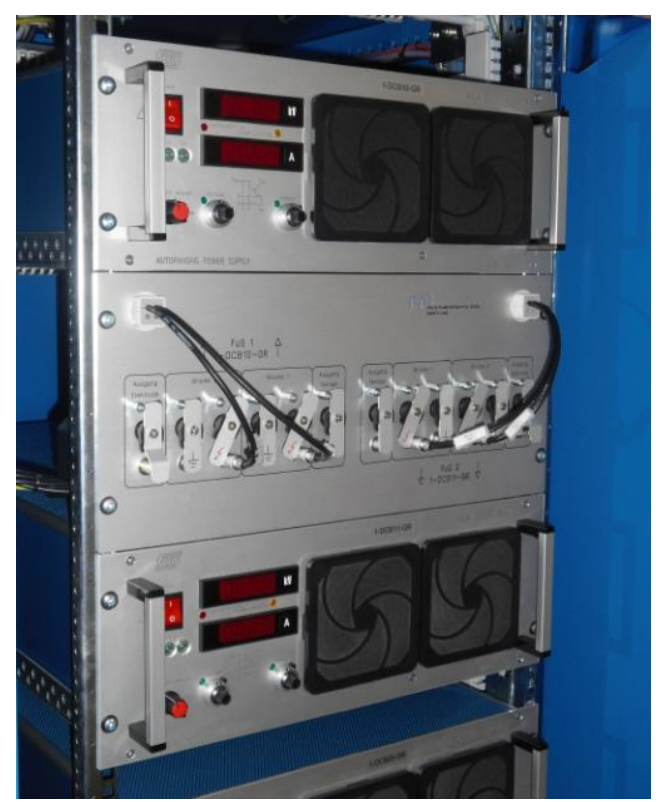

Fig.1 rack showing two converters and the connections to the electrodes, to the test load as well as the grounding bridges.

\subsection{Control system}

The main subject of the control system is the combination of the ten power supplies to a common system, and depending on the requirements the control of the whole system, acting as a slave with respect to the W7-X main control $[2]$.

In principle there are three main functions: the process control, the access control and the operation mode control.

The process control is responsible for the transmission of reference values, actual values and commands from and to the W7-X main control which are related to the glow discharge process.

The access control defines if the glow discharge power supply control acts as a local master or as a slave with respect to the W7-X main control. During the experimental phase of W7-X the so called "subordinated operation" will be the most used method. The W7-X main control acts as a master and all commands, values etc. will be given by the W7-X master. The physical location is always the W7-X main control room. If the glow discharge power supply control acts as the master (so called autonomous operation), then the commands will be given from the local control. It has to be noticed that local control does not necessarily mean that the control has to done directly in front of the system. 
Autonomous operation means only that the whole system runs independently from the W7-X main control. Within the autonomous operation it can be decided if the operator is physically located directly in front of the power supplies (so called autonomous/local) or at another place, connected via Ethernet connection (so called autonomous/remote operation).

The operation mode control is responsible for the correct definition and the correct switch over between the operation modes, listed in table 2. All of the converters are always in the same operation mode. The only exception is the Test mode. One power supply can be operated independently from the other converters and separated from the glow discharge electrodes. This operation mode is necessary, to allow a test of the power supplies independently from the operation of W7-X. But it creates additional effort with respect to the grounding. For normal operation, when the glow discharge electrode is connected to the power supply only one grounding connector is necessary. In the Test mode, both, electrodes and converters have to be electrically separated. Consequently both elements now have to be grounded. The control system is concentrated in one 19 inch rack. It contains the PLC of the Simatic S7-400 type, the input and output cards, the components for the Profibus DP and for the Ethernet connections and an operator panel for the local control.

\subsection{Safety control}

The system operates with a voltage above one $\mathrm{kV}$, according to official safety rules this requires special measures to avoid any injuries of personals. Therefore a separate safety control has to be established. It communicates directly with the W7-X central safety system and is responsible for the generation of the signals which confirm the status of the power supply system and gives certain safety interlock signals depending on the status of the whole W7-X experiment.

One main task is the safe detection of the grounding status of converters and electrodes. Both, converter and electrode are equipped with two independent grounding measures: one grounding switch and in addition a bridge to ground which has to be placed manually. Both grounding measures have to be observed by the safety control unit. Only if both grounding parts are successfully connected, the signal "safe grounded" will be generated. The reason for such extraordinary effort is that in case of a failure in a converter or in the process control and without grounding the whole W7-X could be set at high voltages of up to $3 \mathrm{kV}$, which creates a high danger. For the secure grounding of the power supplies HV output and the electrodes as well as HV contactors with forcibly guided message contacts were specified. Unfortunately such contactors were not available on the market for a voltage of $3 \mathrm{kV}$. As a sufficient alternative a contactor with two mechanically connected two way power contacts working on one coil was identified to fulfill the needs of a save contactor status indication. One set of the power contacts is used for switching the high voltage, the other one is used for the status information. To verify the usability of these contactors additional tests under the specific working conditions and a HV Test with $9 \mathrm{kV}$ between the two contact sets have been performed. The tests showed that one contact can be used as an indication contact while the other contact is on HV potential.

Table 2: operation modes of the glow discharge power supplies

\begin{tabular}{|c|c|c|c|c|c|c|}
\hline \multirow[t]{2}{*}{ Operation mode } & \multirow[t]{2}{*}{$\begin{array}{l}\text { Grid voltage of } \\
\text { the converters }\end{array}$} & \multirow[t]{2}{*}{$\begin{array}{c}\text { Reference } \\
\text { values of the } \\
\text { converters }\end{array}$} & \multicolumn{2}{|c|}{$\begin{array}{l}\text { Grounding of the } \\
\text { converter output }\end{array}$} & \multicolumn{2}{|c|}{$\begin{array}{l}\text { Grounding of the } \\
\text { electrodes }\end{array}$} \\
\hline & & & Relays & manually & Relays & manually \\
\hline OFF & off & Null & $\mathrm{X}$ & $(\mathrm{X})$ & $\mathrm{X}$ & $(\mathrm{X})$ \\
\hline READY & on & Null & $\mathrm{X}$ & & $\mathrm{X}$ & \\
\hline $\mathrm{ON}$ & on & Operator & & & & \\
\hline Test: converter under test & on & Operator & & & $\mathrm{X}$ & $\mathrm{X}$ \\
\hline other converters & off & Null & $\mathrm{X}$ & $\mathrm{X}$ & $X$ & $\mathrm{X}$ \\
\hline Emergency stop & off & Null & $\mathrm{X}$ & $(\mathrm{X})$ & $\mathrm{X}$ & $(\mathrm{X})$ \\
\hline failure & n.d. & n.d. & n.d. & n.d. & n.d. & n.d. \\
\hline
\end{tabular}

Another task of the safety control is the check of the doors of the converter rack as well as of the test load rack in order to avoid the risk of touching parts on high voltage. The doors are electromagnetically blocked and their function will be checked by independent sensors. The check of the status of all breakers, especially the main grid breakers, the generation of messages about emergency shut downs and an optical signalization of the present operation mode on site, are further tasks of the safety control. The safety control is also in charge of receiving the general signal which allows 
the start of the system. A PLC of the Siemens S7-300F type is used in conjunction with the related safety software package. Unless the safety control is situated in the same rack than the normal control, all input and output and transmission components are separated from each other. Also the connection to the W7-X main safety control is done by watchdog observed separate lines. All error messages will be stored in a local storage for later investigation. Depending on the kind of failure, the message has to be confirmed or it is a warning message only.

\subsection{Test load}

The test load gives the opportunity to check the output parameters of a converter which is physically still part of the system, but electrically separated. It is also necessary to carry out the maintenance without powering the electrodes. It allows to operate a converter either at full voltage of $3 \mathrm{kV}$ and $1 \mathrm{~A}$ or at full current of $3 \mathrm{~A}$ at $1 \mathrm{kV}$. Therefore the load is switchable between $3 \mathrm{kOhm}$ and $1 \mathrm{kOhm}$ respectively. Due to the high voltage the load is integrated in a separate and closed rack with an air cooling by fans. Also the test load rack is equipped with safety sensors at the removable parts of the cabinet and the door is electromagnetically blocked as well in order to avoid any danger to the operators due to the high voltage during the test.

\section{Project realizations}

For the project realization the scope of delivery was divided into the procurement of the converters on one hand and the design of the high voltage switch gear, the power distribution unit, the control and safety system and integration of the converters creating power supply units including the design of the racks on the other hand. The procurement of the converters was done by the IPP purchasing department while the remaining part was awarded to the company PulsPlasma-Technik in Dortmund (PPT). The integration of the overall system into the IPP facilities was done by IPP staff. For this a suitable site with a direct connection to the Torus Hall to keep the cableways short needed to be identified. Also the requirement for the cable ways of the high voltage cables under given radiation safety requirements was a limiting factor for the site as special shielding measures have to be taken into account when sending cables through an opening in the wall of the torus hall. The connection to the power of the main and auxiliary supplies coming from the W7-X experimental grid needed to be established as well as the connections to the W7-X main control and the central safety system which were prepared in terms of hard- and software. With these surrounding parameters a design for the power supply system was set up by PPT under the supervision of IPP. Special care has been given to the secure indication of the hardwired grounding switch gear which is the main part of the safety control system. Also the protection of the sensitive parts of the control system against disturbances from the grid was carefully laid out. Under the close collaboration with IPP it was possible to keep the design phase short and to let the manufacturing start very soon as certain parts of the design could be easily fixed and the procurement of the related components was already started while further details of the design like certain safety signals and the realization of the operating principles were still under way.

After the manufacturing of the whole plant a factory test was carried out together with IPP. Besides the normative tests e.g. regarding electrical insulation one focus was the thermal behavior of the cabinets. The one cabinet containing four power supply units was tested under full load (max. current at $1 \mathrm{kV}$ leading to losses of about $2 \mathrm{~kW}$ in the rack) to verify the efficiency of the cooling design. The hot spots inside the cabinet were located and their temperature was observed. The highest temperature was measured at about $34{ }^{\circ} \mathrm{C}$ at the expected hot spot between the two upper power supply units. The functionality of the process control and the safety control together with the operating principles from the local operator panel in autonomous/local operation has been successfully tested.

Afterwards the shipment to the IPP site and the installation of the plant took place which was also part of the work package for PPT. Now connected to the IPP facilities the plant was finally tested in conjunction with the W7-X -grid and -main control, the central safety system and the data acquisition system responsible for collecting the voltage and current values of all ten converters for later evaluation. With one power supply unit connected to the test load the system was operated in autonomous/remote and subordinated control mode. Operating principles, safety interlocking and data acquisition were tested with power. Also the connection of the system to all ten electrodes was simulated to proof the operating principles as well.

Fig. 2 shows the power supply system after the final tests. The control rack is located at the left hand side; the other three racks contain the converters and the grounding elements. All racks are separated from the ground potential by 5 $\mathrm{mm}$ plastic sheets. The resistance to ground was measured to $10 \mathrm{MOhm}$ and will be re-checked regularly during operation. In the final step the routing for the high voltage cables and the design of the radiation shielding for the feed through hole in the Torus hall wall have to be designed. Cabling will consists of one connection to each anode of the electrode and one cable connected to the vessel acting as the cathode for all ten electrodes. Taking into account the fire safety rules for cabling a non halogen RG58 type cable with $0,8 \mathrm{~mm}^{2}$ was chosen for the ten connections to the electrodes consuming a total length of about $300 \mathrm{~m}$. The length for one connection will not exceed $40 \mathrm{~m}$ to keep the voltage drop and the influence of capacitance and inductance on the controller behavior of the converters low. The type of cable necessary for the one connection to the cathode was not available in a non halogen version. Therefore a special 
low halogen type was ordered. This cable has a $6 \mathrm{~mm}^{2}$ cross section to carry the whole current coming from the ten electrodes.

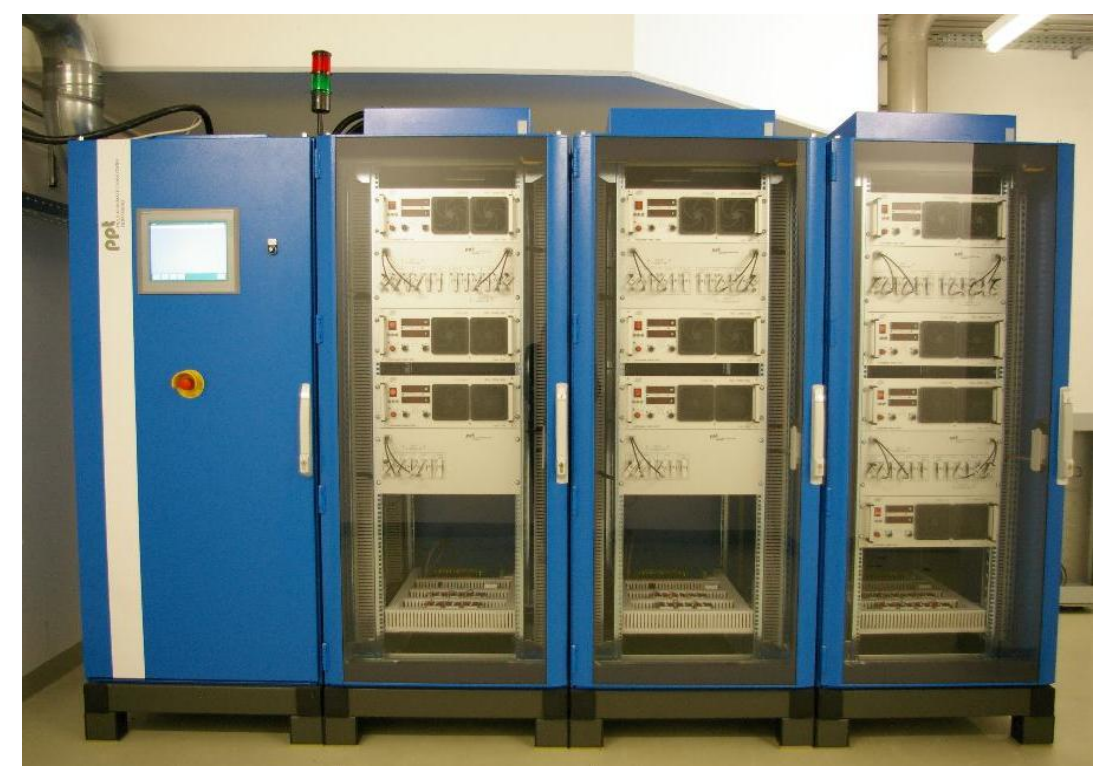

Fig.2 Power supply system after installation.

The design and preparation of the radiation shielding is scheduled for 2011 while the final installation of the cables and the connection to the glow discharge electrodes will take place close to the end of the assembly of W7-X in 2014 . This time will be used to gain first operation experiences for the staff at IPP using the test load.

\section{Summary}

The glow discharge system of Wendelstein 7-X consists of ten converters with maximum rating of $3 \mathrm{kV}$ and $3 \mathrm{~A}$, respectively. A sophisticated process control assisted by a safety control system allows the on-site operation as well as from the W7-X main control room. The safety control prevents the personal from the danger of high voltages. The system was build, installed and successfully tested at the Max-Planck-Institut für Plasmaphysik in Greifwald, Germany in 2010 and is ready for operation tests.

\section{References}

[1] A. Spring, R. Brakel and H. Niedermeyer, Wall conditioning for Wendelstein 7-X by glow discharge, Fusion Engineering and Design 66-68 (2003), 371-375.

[2] J. Schacht, H. Laqua, A. Spring et al., "Task and structure of the WENDELSTEIN 7-X control system", Fusion Engineering and Design 81 (2006), 1799-1806 